\title{
Textlinguistik und Übersetzungswissenschaft - Entwicklung und Interaktion
}

\section{Peter Holzer}

Institut für Translationswissenschaft der Universität Innsbruck

Since the beginning of the 1980s the categories of text linguistics have played an increasingly important role in both theoretical and applied translation studies. They have attracted more and more attention in the course of attempts to establish a translation-oriented text theory and text analysis for teaching purposes. In this context translation studies have been able to make a useful contribution to the linguistic debate on text cohesion and coherence and have in turn greatly benefited from that debate. At the same time, through contact with other disciplines, including the social sciences and cultural sciences, translation studies have helped to generate new aspects in text linguistics and to extend its horizons. Today text linguistics and translation studies exist in a form of symbiosis that has attractive potential for the future.

\section{Einleitung}

Linguistik und Übersetzungswissenschaft stellen auf den ersten Blick zwei Bereiche dar, deren Ähnlichkeit auf der Hand liegt: Bei beiden steht die Sprache als Gegenstand der Betrachtung im Vordergrund, mit dem Unterschied, dass die Linguistik als ursprünglich systembezogene, strukturalistische Sprachwissenschaft und in der Folge als Teildisziplin einer allgemeinen und umfassenden Sprachwissenschaft sich mit der Betrachtung einer einzelnen Sprache oder auch universeller Phänomene befasst, die Übersetzungswissenschaft hingegen den Vorgang des Übertragens von einer Sprache in eine andere bzw. von einer Kultur in eine andere in den Mittelpunkt ihrer Untersuchungen stellt. Bezieht man das Feld der Kontrastiven Linguistik und der damit einhergehenden vergleichenden Untersuchung zweier oder mehrerer Sprachen mit ein, so könnte man annehmen, dass damit eine Annäherung an die Übersetzungswissenschaft beinahe schon vollzogen ist. Und doch ist es von hier noch ein weites Stück bis zu dem, was man im modernen Sinn unter Übersetzungswissenschaft oder Translationswissenschaft - eine Bezeichnung, die im Deutschen als Hyperonym für Übersetzungswissenschaft und Dolmetschwissenschaft immer häufiger Verwendung findet ${ }^{1}$ - versteht.

Genau hier liegt auch das Problem einer Auseinandersetzung, die in den letzten zwei Jahrzehnten zwischen der Linguistik auf der einen und der Übersetzungswissenschaft auf der anderen Seite stattgefunden hat: Die Vertreter eines linguistischen Ansatzes beim Übersetzen beschränkten sich vielfach darauf, im Übersetzen nur einen linguistischen Transfer im Sinne einer Transkodierung von einem System in ein ande- 
res zu sehen. Ein Hauptaugenmerk dabei galt dem Problem der Äquivalenz. Sie übersahen dabei, dass jede Sprache in eine bestimmte Kultur eingebunden ist und dass kulturelle Merkmale mit einer systemhaften Beschreibung nicht so einfach zu fassen sind. Zwar hat die pragmatische Wende hier neue Gesichtspunkte gebracht, eine grundlegende Änderung der Sichtweise war damit aber nicht verknüpft. Als eine Art Gegenpol auf der anderen Seite entwickelte sich ab Mitte der 80er Jahre die sog. funktionalistische Translationswissenschaft und die handlungstheoretisch begründete Translatologie (s. Reiss \& Vermeer 1984, Holz-Mänttäri 1984), die in ihrer Grundausrichtung, aber auch in verschiedensten Abwandlungen bis heute Zuspruch findet. Eine der Grundthesen der funktionalen Translationswissenschaft lautet, der Translationsprozess solle an der zielkulturellen Situation ausgerichtet sein, damit die Übersetzung die gewünschte Funktion erfüllen könne. Die Funktionalisten wehrten sich mit Vehemenz gegen jegliche linguistische Betrachtungsweise und gegen eine Vereinnahmung durch deren Vertreter. Das Ergebnis war ein Aufeinanderprallen von Standpunkten, die zwar letztlich zur Etablierung des Faches Übersetzungswissenschaft als eigenständige Disziplin beigetragen haben, jedoch kaum Argumente für eine wechselseitige Beziehung zwischen der Linguistik samt ihren Teilbereichen und der Übersetzungswissenschaft zuließen. Ich möchte daher im Folgenden versuchen, die Entwicklung beider Disziplinen - innerhalb der Linguistik den der Textlinguistik - kurz zu skizzieren und anhand einiger Punkte darlegen, dass die wechselseitige Beeinflussung zu einer sinnhaften Ergänzung und Vervollständigung der angesprochenen Bereiche geführt hat.

\section{Entwicklungsgeschichtliche Marksteine}

Mitte der 80er Jahre erfährt die bis dahin vorwiegend unter dem Postulat der Äquivalenz geführte Diskussion innerhalb der Übersetzungswissenschaft $^{2}$ im deutschsprachigen Raum eine Wende, deren Kernthesen bis heute fortwirken. Verantwortlich dafür zeichnen vor allem zwei Publikationen: Reiss \& Vermeers Grundlagen einer allgemeinen Translationstheorie (1984) und Snell-Hornbys Übersetzungswissenschaft - eine Neuorientierung (1986). In dem von Reiss \& Vermeer verfassten Werk werden die Grundlagen der Skopostheorie und der damit in Verbindung stehenden funktionalistischen Translationstheorie dargelegt, die bis heute im Zentrum heftiger Diskussionen stehen. Trotz aller Kritik an diesem Ansatz muss man feststellen, dass er der modernen Übersetzungswissenschaft entscheidende Impulse verliehen hat. Das lässt sich auch von anderen, vielfach weiterentwickelten funktionalen Ansätzen (wie etwa bei Nord 1993, 1997) behaupten.

Snell-Hornby (1986:9ff.) plädiert für die Bestimmung eines Forschungsbereichs der Übersetzungswissenschaft als eigenständiger Disziplin anstelle der bislang üblichen Vorstellung, ein Teilgebiet der 
Linguistik $\mathrm{zu}$ sein. Die wissenschaftliche Weiterentwicklung sei durch das klassische Kästchendenken mit strengen Trennungslinien behindert worden. In ihrer konkreten Realisierung im Text ließe sich Sprache nicht ausschließlich auf ein abstraktes Regelsystem reduzieren. So schreibt sie:

An die Stelle der traditionellen Typologien sollten hier Prototypologien treten, die nicht auf Trennung, sondern auf Fokussierung hinzielen und dabei verschwommene Ränder und Mischtypen zulassen. Auch die strenge methodische Isolierung tritt in den Hintergrund: beim Übersetzen geht es nicht um isolierbare Einheiten, sondern um ein Gewebe von Bezügen, wobei die Bedeutung der einzelnen Wörter durch ihre kontextuelle Relevanz und ihre Funktion im Textganzen bedingt ist. (Snell-Hornby 1986:16)

In diesem Sinne entwirft Snell-Hornby, ausgehend von Textsorten und übersetzungsrelevanten Gesichtspunkten ein Schichten- oder Stratifikationsmodell, das sich ohne scharfe Trennungslinie und mit fließenden Übergängen von der Makroebene bis zur Mikroebene bewegt. In der von ihr benannten Ebene $\mathrm{E}$ sind die übersetzungsrelevanten Bereiche der Linguistik angeführt:

Grundlegend für sämtliches Übersetzen ist die Textlinguistik in allen Aspekten, von der Analyse der Makrostruktur über die Thema-RhemaGliederung und die Satzperspektive bis zur Kohärenz und Kohäsion. (Snell-Hornby 1986:19f.)

Der Vorstellung einer Prototypologie entspricht die interdisziplinäre Offenheit und das Integrieren verschiedener Methoden der Textbeschreibung. In diesem Sinne hat Snell-Hornby nicht nur für eine offenere, flexiblere Konzeption des Übersetzens plädiert, sie hat auch die Brücke zur Textlinguistik geschlagen und zudem mit dem Begriff des Prototypen eine Entwicklung innerhalb der Semantik vorweggenommen.

\subsection{Textlinguistik im Dienste der Übersetzungswissenschaft}

Bereits in den 70er Jahren wandte sich die Linguistik verstärkt satzübergreifenden Strukturen $\mathrm{zu}$, es entstand die Textlinguistik. Wolfgang Dressler, einer der Begründer der Textlinguistik, knüpfte in seinem Aufsatz Der Beitrag der Textlinguistik zur Übersetzungswissenschaft (1974) beim Thema (Un)übersetzbarkeit an und stellte fest, dass dieses durch das Auftreten der Textlinguistik in ein neues Stadium getreten sei. Die Textlinguistik als Wissenschaft von der sprachlichen Konstitution von Texten, habe die Satzgrenze überschritten, um transphrastische Strukturen innerhalb der Texte zu untersuchen. Ein einzelner, aus dem Textzusammenhang gerissener Satz sei deshalb als unvollständig zu betrachten und stelle keine unabhängige Einheit dar. Daraus ergibt sich 
für Dressler die Schlussfolgerung, dass eine vollständige, eindeutige (d.h. symmetrische) Übersetzungsäquivalenz nicht existiere und es damit auch keine vollständige Übersetzbarkeit gebe (Dressler 1974:62). Die weiteren Ausführungen dieses Aufsatzes klingen wie ein Programm zur textlinguistischen Fundierung der Translationswissenschaft. Durch die Untersuchung von Textsorten könnten Texte effizienter dekodiert und besser auf die Erwartungshaltungen der Adressaten eingestellt werden. Bei der Textpragmatik sei auf die Funktion von Sprechakten bzw. Sprechhandlungen $\mathrm{zu}$ achten.

Nach Dressler war es Eugenio Coseriu, ebenfalls ein prominenter Vertreter der Textlinguistik, der den kontrastivlinguistischen Zugang zum Problem des Übersetzens in Frage stellte. In seinem Aufsatz Falsche und richtige Fragestellungen in der Übersetzungstheorie (1981) postulierte er explizit das finalistische Prinzip der Übersetzung. Seine Kritik an einem einfachen kontrastiven Zugang zum Problem des Übersetzens setzt bei der Dichotomie zwischen langue und parole an. Eine der falschen Erwartungen, die man gegenüber der Übersetzung hege, sei es, dass eine Übersetzung einer einfachen Ersetzung gleichkäme. Man dürfe nicht fragen, welches Element des jeweils anderen Sprachsystems äquivalent sei, sondern vielmehr "wie nennt man den gleichen Sachverhalt bzw. Tatbestand in einer anderen Sprache in der gleichen Situation" (Coseriu 1981:33). Im Gegensatz zur theoretischen Unmöglichkeit der Übersetzung auf der Wortund Satzebene beweise die erfolgreiche Übersetzungspraxis, dass Übersetzungen sehr wohl möglich sind, wenn man vom Textganzen ausgeht. Auf der Ebene des Textganzen löse sich der Widerspruch zwischen der theoretischen Unmöglichkeit des Übersetzens und der erfolgreichen Übersetzungspraxis auf.

Sowohl Dressler als auch Coseriu hatten ihren Blick auf die um eine pragmatische Komponente erweiterte Ausprägung der Textlinguistik gerichtet und damit diese für die Übersetzungswissenschaft brauchbar gemacht. Die früheren, stärker strukturalistisch orientierten textlinguistischen Arbeiten sind von der Übersetzungswissenschaft nicht aufgegriffen worden. Die Erkenntnis, dass die Bedeutung eines Textes und damit auch der textkonstituierenden Verfahren nicht ohne Berücksichtigung der situativen Faktoren zu bestimmen sind, und die Herausarbeitung der Interdependenz zwischen textexternen und textinternen Faktoren, die letztlich zu einer Textfunktionslinguistik und zu verschiedenen Texttypologien führte, hat die Entwicklung der modernen Übersetzungswissenschaft stark beeinflusst.

\subsection{Textlinguistik und Ausgangstextanalyse}

Arbeiten, bei denen die Übersetzungsdidaktik im Mittelpunkt stand, trugen mit auch zur Rezeption textlinguistischer Methoden und Erkenntnisse bei. Hervorzuheben sind hier im Besonderen Reiss (1976), Hönig \& Kußmaul (1982) und Nord (1988). Der Text wurde dabei zunehmend als 
Funktionseinheit in den Blickpunkt gerückt: In der Analysephase, in der der Ausgangstext (AT) rezipiert und verstanden werden muss, in der Synthesesphase, in der der Zieltext (ZT) formuliert werden muss, und in der Evaluierungsphase, in der das Produkt Übersetzung an den Vorgaben des Übersetzungsauftrags gemessen werden muss. Schon Reiss (1976) weist mit Nachdruck auf die Tatsache hin, dass eine gründliche Analyse des AT eine unabdingbare Voraussetzung für das Übersetzen darstellt. Ansätze zur Methodik einer solchen Analyse wurden ab Mitte der 70er Jahre entwickelt also etwa zeitgleich zur pragmatischen Wende in der Textlinguistik. Dabei hängt es von der übersetzungstheoretischen Ausrichtung ab, ob diese Analyse nur zur Verständnissicherung oder bereits zur Bereitstellung von Übersetzungseinheiten dienen soll. Äquivalenzorientierte Ansätze bevorzugen eine sprachenpaarspezifische AT-Analyse, hermeneutische Ansätze (so etwa Stolze 1982) nutzen textlinguistische Verfahren zur Vertiefung des intuitiven Textverständnisses. Funktionale Ansätze stellen dagegen die pragmatischen Aspekte des interkulturellen Kommunikationsaktes in den Vordergrund, so etwa Hönig (1986) und Nord (1988).'

Die meisten texttheoretisch begründeten Modelle der übersetzungsrelevanten Textanalyse orientieren sich an den W-Fragen der sog. LasswellFormel: Wer sagt was zu wem wann wo wie und zu welchem Zweck? Textlinguistische Kategorien im engeren Sinn, die hier nutzbar gemacht werden, betreffen vornehmlich die Bereiche Thematik, Inhalt und Textstruktur, wie z.B. Thema-Rhema-Gliederung und thematische Progression, funktionale Satzperspektive, kohärenz- und kohäsionsstiftende Mittel wie Anapher/Katapher, Rekurrenz, Isotopie, Substitutionsmechanismen oder Konnektoren.

Dass das aus einem Übersetzungsprozess resultierende Produkt ein Text sein sollte, der den Anforderungen der zielkulturellen Kommunikationsgemeinschaft im Allgemeinen und Exemplaren bestimmter Textsorten im Besonderen genügt, ist inzwischen zur Binsenweisheit geworden. In der übersetzungswissenschaftlichen Literatur wird diese Forderung besonders von den Vertretern funktionaler Ansätze erhoben - wie sie zu verwirklichen ist und wie textlinguistische Methoden dazu beitragen können, wird allerdings selten explizit dargestellt. Dass Texte auf die Bedürfnisse und Erwartungen der Adressaten zugeschnitten sein müssen, bleibt eine leere Formel, solange die zielkulturellen Vertextungsgewohnheiten nicht durch umfangreiche Korpora belegt sind.

An dieser Stelle noch ein Wort zum Thema Textsorte: Die translationsrelevante Textsortenproblematik wurde - wie allgemein bekannt - bereits 1976 von Katharina Reiss in ihrem viel zitierten Texttypenmodell gebündelt, das in mehreren Modifikationen zum Klassiker der Übersetzungsdidaktik wurde. Das englische Pendant dazu findet sich bei Newmark (1995), der das Spektrum der Texttypen unter Berücksichtigung der phatischen und metalingualen Funktion etwas erweitert darstellte (1995:40). Das vermehrte Aufkommen von Fachtexten in der übersetzerischen Praxis hat dazu geführt, dass speziell im Bereich der Naturwissenschaften und der Technik Subtypologisierungen des Reissschen informati- 
ven Texttyps vorgenommen wurden. Erwähnenswert hierzu ist Göpferichs Textsorten in Naturwissenschaft und Technik (1995).

\section{Textlinguistische Kategorien im Dienst der Übersetzung}

Textlinguistische Kategorien haben - wie schon oben erwähnt - seit Beginn der 80er Jahre im Bereich der Übersetzungswissenschaft, im Besonderen im Bereich der Angewandten Übersetzungswissenschaft, zunehmend an Bedeutung gewonnen. Sie sind im Zuge der Bemühungen um eine sowohl für die Lehre als auch für die Praxis brauchbare übersetzungsbezogene Textanalyse immer stärker ins Bewusstsein getreten (s. dazu Holzer 1998a, 1998b). Die Übersetzungswissenschaft konnte sich hier an die linguistische Diskussion über Textkohäsion und Textkohärenz gewissermaßen anhängen und letztlich wohl auch erheblich davon profitieren. Sie hat deren Schwerpunktbildung zunächst in der Textgrammatik - hier allerdings mit großen Einschränkungen -, dann in der Textsemantik und schließlich in der Textpragmatik mitvollzogen und versucht, aus jeder der drei Textdimensionen Ansätze für die Methodik einer übersetzungsbezogenen Textanalyse abzuleiten. Der enge Kontakt mit der Textlinguistik, aber auch mit bestimmten Bereichen der sozialwissenschaftlichen Forschung, z.B. der Handlungstheorie, hat für die Übersetzungswissenschaft neue Erkenntnisse gebracht: So hat sich das heute selbstverständliche Wissen herausgebildet, dass u.a. die Einbettung des Textgegenstandes in eine Kommunikationssituation, die Intention des Textproduzenten, die Kenntnis des Textempfängers theoretisch und praktisch in den Übersetzungsprozess einbezogen werden müssen.

Es ist heute auch unbestritten, dass Analysekategorien nicht auf einer Ebene anzusetzen sind, sondern dass sie eine Hierarchie bilden und dabei den pragmatischen Faktoren meist ein hoher Stellenwert eingeräumt wird: Durch die pragmatischen Kategorien werden die Rahmenbedingungen für die semantischen und syntaktischen Gegebenheiten des Textes hergestellt. Diese Rahmenbedingungen, die auf der Beziehung zwischen Autor, Text und Empfänger basieren, kommen letzten Endes in der Textfunktion zum Ausdruck.

\subsection{Isotopie als Instrument der Übersetzungs-Evaluation}

Ein besonderes Augenmerk beim Einsatz textlinguistischer Instrumentarien verdienen die mit Wort- und Textsemantik verbundenen Konzepte, insbesondere das Isotopie-Konzept, das im Rahmen der Textlinguistik zwar seinen Niederschlag gefunden hat, innerhalb der Übersetzungswissenschaft jedoch weitgehend vernachlässigt wurde ${ }^{3}$. Thiel (1996:62) meint, dass dies angesichts der nicht vorhandenen Breitenwirkung in der textlinguistischen Literatur nicht verwunderlich sei und zumindest Hinweise in Publikationen zu erwarten wären, die generell in Problemstellungen des Übersetzens einführen. Dieser Meinung kann man zustimmen, obgleich die Frage eines kau- 
salen Zusammenhangs zwischen der Breitenwirkung in der textlinguistischen Literatur auf der einen Seite und in der übersetzungswissenschaftlichen Literatur auf der anderen Seite noch zu diskutieren wäre.

Der Begriff Isotopie geht auf den französischen Sprachwissenschafter Greimas zurück, der ihn in seinem viel zitierten Buch Sémantique structurale (1966) eingehend erläutert ${ }^{4}$. In der deutschsprachigen textlinguistischen Literatur erscheint der Begriff ab den frühen 70er Jahren und wird dort als Merkmalrekurrenz im Text beschrieben (vgl. z.B. Kallmeyer et al. 1974:147). Isotopie versteht sich danach als kohärenzstiftender und textkonstitutiver Bedeutungszusammenhang von aufeinander folgenden Lexemen aufgrund gemeinsamer semantischer Merkmale. Lexeme, die in einem Text über ein gemeinsames rekurrent-dominantes semantisches Merkmal verknüpft sind, konstituieren eine Isotopieebene. Eine solche Ebene kann immer dann angenommen werden, wenn wenigstens zwei Lexeme die Verknüpfung durch ein gemeinsames semantisches Merkmal aufweisen.

Eine Isotopieebene kann sich über größere Textpassagen erstrecken, gegebenenfalls auch über einen ganzen Text, im Maximalfall gehören alle Lexeme eines Textes zu einer einzigen Isotopieebene. Als Beispiel kann hier die Textsorte Wetterbericht angeführt werden, wo ein überwiegender Teil der Lexeme das Merkmal [meteorologisch] aufweist. Meist sind es jedoch mehrere Ebenen, die in einem hierarchischen Verhältnis zueinander stehen.

Isotopie ist bei Greimas ein Instrument der semiotischen Textanalyse. Vorrangiges Ziel dabei ist, die Organisation der Bedeutung als solcher im Textablauf herauszufinden. Die so verstandene semiotisch-orientierte Analyse integriert Sichtweisen, die die Isotopie als eine Textualisierung des Wortfeldbegriffs interpretieren, insofern die lexikalischen Elemente eines Textes thematisch bedingte Wortnetze bilden (s. dazu auch Weinrich 1976:14ff.). Nicht umsonst wurde die Isotopie in ihrer Grundkonzeption vorwiegend zur Analyse und Interpretation literarischästhetischer Texte verwendet, bei denen mitunter durch eine Zerstörung syntaktischer Bezüge und die Durchbrechung von Textmustern das Textverstehen erschwert wird. So hat z.B. Poulsen in seinem Aufsatz Textlinguistik und Übersetzungskritik (1981) die Isotopie für die Evaluierung von Übersetzungen nutzbar gemacht. Am Beispiel des Steppenwolf-Gedichts von Hermann Hesse aus dem Deutschen ins Dänische und ins Englische wird aufgezeigt, dass Isotopieebenen als potentielle Übersetzungseinheiten (1981:303) zu betrachten sind und dass der Übersetzungskritiker mit dem Vergleich der Konstituenten der Isotopieebenen im ausgangssprachlichen Text und deren Wiedergabe im zielsprachlichen Text ein Instrument für die Beurteilung der übersetzerischen Leistung in der Hand hat. Diesem Argument kann man sich nur voll und ganz anschließen.

Aus der eigenen Erfahrung im Übersetzungsunterricht lassen sich folgende Punkte anführen, die für eine Isotopie-Analyse sprechen:

- Die Analyse der Isotopieebenen im AT führt zur Beschreibung der Bedeutungsstruktur als der semantischen Textkonstante. Das 
Textganze wird in seinen semantischen Grundzusammenhängen überschaubar gemacht; daraus ergibt sich die Möglichkeit, strukturell begründete Kohärenzforderungen für den $\mathrm{ZT}$ zu formulieren und übersetzerische Entscheidungen konkret vorzubereiten.

- Das Translat kann durch einen Vergleich der Isotopiestränge in AT und ZT auf seine intratextuelle Kohärenz und semantisch-pragmatische Stimmigkeit ${ }^{5}$ überprüft werden.

- Isotopieebenen sind aussagekräftig in Bezug auf Thematik und Funktion des Textes.

- Mit Hilfe der Isotopie wird eine Verständnissicherung im Übersetzungsprozess ermöglicht. Darüber hinaus lassen sich die wesentlichen Informationen eines Textes überindividuell nachvollziehbar analysieren.

- Isotopieebenen sind als potentielle Übersetzungseinheiten zu betrachten und stellen vor allem für den Übersetzungskritiker objektive Bewertungskriterien dar.

\subsection{Das Konzept der scenes and frames}

Wenn man von semantischen Konzepten und deren Anwendbarkeit im Übersetzungsprozess spricht, drängen sich natürlich auch kognitive Ansätze auf, die in den letzten Jahren Eingang in die wissenschaftliche Literatur gefunden haben. Am bekanntesten ist dabei wohl das Konzept der scenes and frames, dessen Ausgangspunkt Fillmores viel zitierter Aufsatz scenesand-frames semantics (1977) darstellt ${ }^{6}$. Ein Vergleich des oben erwähnten Isotopie-Konzepts mit dem Konzept des scenes and frames liegt nicht von vornherein auf der Hand: Ersteres hat den Schwerpunkt in der kohärenzorientierten Textanalyse und in der Textverknüpfung, letzteres im Verstehensprozess und den Wissenskomponenten, die beim Gebrauch von Lexemen aktiviert werden. Beide setzen jedoch auf der Wortebene an und treffen sich somit bei der prinzipiellen Frage nach der Beschreibbarkeit der Wortbedeutung. Mit dem Konzept der scenes and frames wird jedoch die außersprachliche Realität stärker einbezogen. In Anlehnung an die Prototypensemantik ergibt sich die Bedeutung eines Wortes nicht aus der Addition von Merkmalen, sondern baut sich aus den Erfahrungen des Sprechers auf: Der Sprecher findet danach - analog zum Spracherwerb des Kindes - zuerst mittels eigener Erfahrung Zugang zu einer linguistischen Form (frame) und verbindet diese mit einer erlebten Situation (scene). $\mathrm{Zu}$ einem frame gehören somit Lexeme, die auf eine typische komplexe Situation oder scene Bezug nehmen: Wörter wie kaufen, Verkäufer, Kunde heben jeweils einen Aspekt einer bestimmten Situation hervor. In der alltäglichen Kommunikation kann ein Sprecher normalerweise davon ausgehen, dass sein Hörer mit der Kenntnis eines Wortes auch das Wissen über die gesamte komplexe Situation verbindet.

Das Konzept lässt sich auch bei der Textanalyse anwenden und bietet insofern Vorteile gegenüber dem vorhin beschriebenen Konzept der 
Isotopie, als hierbei ein dynamischerer Aspekt der Textassimilation zum Tragen kommt. Die Kohärenz eines Textes ergibt sich aus den einzelnen scenes, die sich im Textverlauf $\mathrm{zu}$ einer komplexen, kommunikativen Gesamt-scene verbinden. Für den Kommunikationsvorgang ist entscheidend, dass die vom Sprecher vorgenommene linguistische Auswahl beim Leser scenes aktiviert und dass dadurch auch die Verwendung weiterer frames im Textverlauf bedingt ist. In Anwendung auf die Translation bedeutet dies, dass der Übersetzer die hinter den frames stehenden scenes in der Ausgangssprache erfassen und verstehen und dazu die passenden frames in der Zielsprache suchen muss, die wiederum die gewünschten scenes beim Adressaten hervorrufen.

Zur Beschreibung des Erfassens und Verstehens szenischer Vorstellungen haben die in der Psycholinguistik geprägten Begriffe bottom-up und top-down (s. dazu Hörmann 1981:124) auch in der Übersetzungswissenschaft inzwischen ihren festen Platz eingenommen. Gemeint ist damit, dass das Verstehen aus einer Interaktion besteht zwischen dem, was als Input von draußen hereinkommt (bottom-up) und dem, was der Hörer/Leser aufgrund seines Wissens und seiner Erwartungen auf die Äußerung projiziert (top-down). In Anwendung auf das Konzept der scenes and frames bedeutet dies, dass die szenische Vorstellung sich als top-down-Wissen niederschlägt. Die Bewusstmachung dieser Verstehensprozesse hat sich besonders im Bereich der Übersetzungsdidaktik niedergeschlagen und darüber hinaus das gesamte Spektrum der Übersetzungswissenschaft nachhaltig beeinflusst.

Die psychologische Sichtweise bringt den Vorteil, dass die Bedeutung eines sprachlichen Ausdrucks nicht mehr analytisch als Summe von Teilen (d.h. von semantischen Merkmalen) gesehen wird, sondern als ein Ganzes, in dem Erfahrung, Wissen etc. von vornherein einen festen Platz einnehmen. Textkohärenz stellt sich damit nicht bloß als eine Verknüpfung von Sprachlichem im engeren Sinn dar: Sie beruht vielmehr auf Operationen der Textproduzenten und -rezipienten, die ihr Wissen auf die einzelnen Textelemente anwenden und so erschließen können, welche Bedeutung und Funktion sie für die Konstitution eines Textinhalts - und damit auch einer Textwelt - haben. Die Aktivierung der Textwelt ergibt sich aus dem Zusammenhang der im Text gegebenen sprachlichen Formen und den damit verbundenen, erlebten Situationen. Für den Übersetzer bedeutet es, dass er die Textwelt des AT aufgrund seiner Erfahrung, seines Sach- und Weltwissens erschließen muss und diese im ZT mit den sprachlichen Mitteln wieder so aufbauen muss, dass sie vom Zieltextrezipienten mit der gewünschten Wirkung aufgenommen wird.

Die Bedeutung eines Ausdrucks, eines Textteils oder eines ganzen Textes wäre dann das Potential, eine gezielte Aktivierung von Wissen durch Textrezipienten hervorzurufen. Da die Sprachzeichen nicht mehr als statische Gegebenheiten aufzufassen sind, können sie besser dem Text angepasst werden: Sie werden zu Zeichen-in-Funktion. Die Anpassungsfähigkeit führt dazu, dass im Prozess der Textrezeption kein gesichertes Bedeutungswissen einfach abgerufen wird, sondern dass ein Zeichen nur dann 
ein bestimmtes Wissen einschließt, wenn es in einer Textwelt aktualisiert wird.

\section{Der Faktor Kultur}

Der eben genannte Ansatz der scenes and frames rückt mit der Einbeziehung des Erfahrungswissens, des Sach- und Weltwissens den Faktor Kultur wesentlich stärker in den Vordergrund. Diese Fokussierung geht wiederum $\mathrm{zu}$ einem guten Teil auf die schon erwähnte funktionale Translationstheorie zurück, sie hat aber bis zu einem gewissen Grad auch einen Einfluss auf die Linguistik, im Besonderen auch auf die Textlinguistik ausgeübt. Die Funktionalisten stützen sich neben ihrer handlungstheoretischen Fundierung auf einen kulturtheoretischen Hintergrund und definieren Translation als transkulturelle Interaktion. Kultur wird dabei als menschliches Gesamtverhalten verstanden. Diese kulturintensive Einbettung der Theorie und damit die Ausweitung der Grundlagen übersetzungswissenschaftlicher Theorienbildung auf den Bereich des menschlichen Gesamthandelns über Kulturgrenzen hinweg stellte bereits in den 80er Jahren eine grundsätzliche Neuorientierung (s. dazu Snell-Hornby 1986) dar. Inzwischen wird die Auffassung von Übersetzen als interkultureller Transferhandlung auch anderen Theorieansätzen zugrunde gelegt (vgl. Nord 1993, 1997).

Die Diskussion von Kultur als Bestandteil der Textwelt, z.B. bei der Untersuchung literarischer Übersetzungen, bedeutet häufig die Analyse ausgangssprachlicher Wörter, Satz- oder Textteile im Hinblick auf ihre mehr oder weniger kulturspezifisch geprägten Bedeutungsinhalte und, im Anschluss daran, die Betrachtung der potentiellen oder bereits realisierten Übersetzungsmöglichkeiten für diese festgestellten kulturellen Inhalte. Die zugrunde liegende Basishypothese dabei lautet, dass kulturelle und nicht lediglich sprachliche Unterschiede das zentrale Problem einer interkulturellen Kommunikation darstellten. Eben diese Erkenntnis hat nicht zuletzt auch Eingang gefunden in eine kommunikationsorientierte, pragmatisch ausgerichtete Textlinguistik, die den Text als sprachliche Handlung betrachtet.

\section{Schlussbemerkung}

Die Beziehungen und Interaktionsebenen zwischen Textlinguistik und Übersetzungswissenschaft sind vielfältig und geben zugleich ein Spiegelbild zweier an Bedeutung zunehmender Bereiche ab. Chronologisch bzw. entwicklungshistorisch betrachtet lassen sich Parallelen feststellen, die mit dem Schlagwort Neuorientierung, vollzogen in den späten 70er und den 80er Jahren, wiederzugeben sind. Diese Neuorientierungen führten dazu, dass auf der einen Seite die Textlinguistik als brauchbares Instrumentarium einer sich emanzipierenden Übersetzungswissenschaft entdeckt wurde, auf 
der anderen Seite die Übersetzungswissenschaft Impulse setzte, die innerhalb der Textlinguistik ihren Niederschlag fanden. Dadurch stellte sich ein Verhältnis der Interdisziplinarität ein, das beiden zugute kommt und bis heute andauert. Konkrete Beispiele lassen sich u.a. im Bereich der Textanalyse und Übersetzungskritik für den Einfluss der Textlinguistik auf die Angewandte Übersetzungswissenschaft, besonders die Übersetzungsdidaktik, finden. Der Beitrag der Übersetzungswissenschaft für die Textlinguistik, bislang noch wenig hinterfragt, besteht vorwiegend in der Fokussierung interkultureller Fragestellungen, die aus intrakultureller Sicht nicht in den Blick kommen. Die Forschungen in diesem Bereich lassen für die nächsten Jahre noch einiges erwarten.

\section{Bibliographie}

Coseriu, Eugenio (1981). "Falsche und richtige Fragestellungen in der Übersetzungstheorie." Wolfram Wilss (Hrsg.) (1981). Übersetzungswissenschaft. Darmstadt: Wissenschaftliche Buchgesellschaft, 27-47.

Dressler, Wolfgang (1974). "Der Beitrag der Textlinguistik zur Übersetzungswissenschaft." Volker Kapp (Hrsg.) (1974). Übersetzer und Dolmetscher. Theoretische Grundlagen, Ausbildung, Berufspraxis. Heidelberg: Quelle \& Meyer, 61-71.

Fillmore, Charles J. (1977). "Scenes-and-frames semantics". A. Zampolli (Hrsg.) (1977). Linguistic Structures Processing. Amsterdam: North Holland, 55-81.

Göpferich, Susanne (1995). Textsorten in Naturwissenschaften und Technik. Pragmatische Typologie - Kontrastierung - Translation. Tübingen: Narr.

Greimas, Algirdas Julien (1966). Sémantique structurale. Recherche de méthode. Paris: Presses Universitaires de France.

Holzer, Peter (1998a). "Isotopie oder 'scenes and frames'." Peter Holzer \& Cornelia Feyrer (Hrsg.) (1998). Text, Sprache, Kultur. Frankfurt am Main: Lang, 159172.

Holzer, Peter (1998b). "Textlinguistische Kategorien und Übersetzen: Isotopie als Instrument der Übersetzungs-Evaluation.” Moderne Sprachen 42(1), 40-51.

Holz-Mänttäri, Justa (1984). Translatorisches Handeln. Helsinki: Finnische Akademie der Wissenschaften.

Hönig, Hans G. \& Paul Kußmaul (1982). Strategie der Übersetzung. Tübingen: Narr.

Hönig, Hans G. (1986). “Übersetzen zwischen Reflex und Reflektion - ein Modell der übersetzungsrelevanten Textanalyse.” Snell-Hornby (1986), 230-252.

Hörmann, Hans (1981). Einführung in die Psycholinguistik. Darmstadt: Wissenschaftliche Buchgesellschaft.

Kallmeyer, Werner et al. (Hrsg.) (1974). Lektürekolleg zur Textlinguistik. Frankfurt a. M.: Athenäum.

Kußmaul, Paul (1996). "Die Bedeutung des Verstehensprozesses für das Übersetzen.” Angelika Lauer et al. (Hrsg.) (1996). Übersetzungswissenschaft im Umbruch. Tübingen: Narr, 229-238.

Newmark, Peter (1995). A Textbook of Translation. New York et al.: Phoenix ELT.

Nord, Christiane (1988). Textanalyse und Übersetzen. Theoretische Grundlagen, Methode und didaktische Anwendung einer übersetzungsrelevanten Textanalyse. Heidelberg: Groos.

Nord, Christiane (1993). Einführung in das funktionale Übersetzen. Am Beispiel von Titeln und Überschriften. Tübingen: Francke. 
Nord, Christiane (1997). Translating as a Purposeful Activity. Functionalist Approaches Explained. Manchester: St. Jerome.

Poulsen, Sven-Olaf (1981). "Textlinguistik und Übersetzungskritik." W. Kühlwein et al. (Hrsg.) (1981). Kontrastive Linguistik und Übersetzungswissenschaft. München: Fink, 300-310.

Rastier, François (1974). "Systematik der Isotopien". Kallmeyer et al. (1974), 153-190.

Rastier, François (1987). Sémantique interprétative. Paris: Presses Universitaires de France.

Reiss, Katharina (1976). Texttyp und Übersetzungsmethode. Der operative Text. Heidelberg: Groos.

Reiss, Katharina \& Hans Vermeer (1984). Grundlegung einer allgemeinen Translationstheorie. Tübingen: Niemeyer.

Snell-Hornby, Mary (Hrsg.) (1986). Übersetzungswissenschaft - eine Neuorientierung. Tübingen: Francke.

Stolze, Radegundis (1982). Grundlagen der Textübersetzung. Heidelberg: Groos.

Stolze, Radegundis (1992). Hermeneutisches Übersetzen. Linguistische Kategorien des Verstehens und Formulierens beim Übersetzen. Tübingen: Narr.

Thiel, Gisela (1996). "Isotopie. Eine textlinguistische Kategorie im Dienst der Übersetzung.” Angelika Lauer et al. (Hrsg.) (1996). Übersetzungswissenschaft im Umbruch. Tübingen: Francke, 59-68.

Vannerem, Mia \& Mary Snell-Hornby (1986). "Die Szene hinter dem Text: 'scenesand-frames-semantics' in der Übersetzung.” Snell-Hornby (1986), 184-206.

Weinrich, Harald (1976). Sprache in Texten. Stuttgart: Klett.

${ }^{1}$ Ich verwende im Folgenden den Ausdruck Übersetzungswissenschaft, zumal die Dolmetschwissenschaft hier nicht Gegenstand der Betrachtung ist.

${ }^{2}$ Streng betrachtet kann man zu diesem Zeitpunkt noch nicht von Übersetzungswissenschaft sprechen, da die Disziplin als solche sich erst in den Folgejahren endgültig etabliert hat.

${ }^{3}$ Ausnahmen bilden Poulsen (1981), Stolze (1982) und Thiel (1996).

${ }^{4}$ Eine detaillierte Behandlung des Isotopie-Konzepts samt Klassifizierung nach bestimmten Typen findet sich u.a. bei Rastier (1974, 1987).

${ }^{5}$ Stimmigkeit ist hier als Sinneinheit zwischen AT und ZT zu verstehen. Vgl. dazu auch Stolze (1992:71ff.).

${ }^{6}$ Der entscheidende Anstoß, die scenes-and-frames-Semantik für das Übersetzen nutzbar zu machen, geht von Vannerem \& Snell-Hornby (1986) aus. 